\title{
Historical Proxy
}

\author{
Marcello Maviglia ${ }^{1}$ \\ 1 University of New Mexico
}

Potential competing interests: The author(s) declared that no potential competing interests exist.

Historical proxy is a concept that provides a framework for understanding the transmission of social traumatic events like Historical Trauma, across community members who have not experienced in person the traumatic events. The theory suggests that Historical Trauma is communicated to others thru the venue of Historical Proxy, defined as a symbolic representation of traumatic experiences which are communicated to individuals and communities in both explicit and subliminal ways.

In other words, the symbolic representation of a traumatic event can be provided by the explicit narrative in a tale, novel, movie etc., and/or be assimilated by individuals and communities by exposure to symbols related to traumatic events (i.e. ruins, monuments, current social issues, psychological and physical distress).

Arguably, the concept of Historical Proxy helps with bridging the gap between historical traumatic events and the emotional distress in individuals who have not personally witnessed and experienced them, but have been exposed to explicit, less explicit, and subliminal symbols representing them.

Moreover, the concept of Historical Proxy, can be viewed both separately from, and in the context of epigenetic theories, which claim that the transmission of traumatic events happens as a consequence of the effects of trauma on the epigenetic make up of individuals, causing negative intergenerational psychological distress. Since the concept is at its beginning stage of development, it will be probably be periodically updated on this page. 